\title{
Identification of inorganic improvised explosive devices by analysis of post-blast residues using portable capillary electrophoresis instrumentation and indirect photometric detection with a light-emitting diode
}

J oseph P. Hutchinson, Christopher J. Evenhuis, Cameron J ohns, Arta ches A. Kazarian, Michael C. Breadmore, Miroslav Mackaa, Emily F. Hilder, Rosanne M. Guijt, Greg W. Dic inoski and Paul R. Haddad*

Australian Centre for Research on Separation Science (ACROSS),

School of Chemistry, Faculty of Science, Engineering and Technology, University of Tasmania, Private Bag 75, Hobart, Tasmania, 7001, Australia

Figure S- 1

Figure S- 2

Figure S- 3

Figure S- 4

Figure S- 5

Figure S- 6

Figure S- 7

Figure S- 8

Figure S- 9 


\section{Figure captions:}

Figure S- 1 Analysis of anions extracted from post-blast residue resulting from a commercial Ammonium Nitrate/Fuel Oil (ANFO) explosive device.

Conditions: The post-blast residue aqueous sa mple was injected without dilution. The capillary electrophoresis method used is outlined in Figure 2.

Figure S- 2 Analysis of cations extracted from post-blast residue resulting from a commercial Ammonium Nitrate/Fuel Oil (ANFO) explosive device.

Conditions: The post-blast residue aqueous sa mple was injected without dilution. The capillary electrophoresis method used is outlined in Figure 3.

Figure S- 3 Analysis of anions extracted from post-blast residues resulting from commercial and improvised Black Powder explosive devices. Black Powder explosive devices typically consist of potassium nitrate, sulphur and charcoal.

Conditions: The post-blast residue aqueous samples were injected without dilution. The capillary electrophoresis method used is outlined in Figure 2.

Figure S- 4 Analysis of cations extracted from post-blast residues resulting from commercial and improvised Black Powder explosive devices.

Conditions: The post-blast residue aqueous samples were injected without dilution. The capillary electrophoresis method used is outlined in Figure 3.

Figure S- 5 Analysis of anions extracted from post-blast residues resulting from an improvised explosive mixture containing chlorate, perchlorate and sugar. Specifically, the inorganic salts used in the composition of the explosive were potassium chlorate/potassium perchlorate. 
Conditions: The post-blast residue aqueous sa mple was injected without dilution. The capillary electrophoresis method used is outlined in Figure 2.

Figure S- 6 Analysis of cations extracted from post-blast residues resulting from an improvised explosive mixture containing chlorate, perchlorate and sugar.

Conditions: The post-blast residue aqueous sa mple was injected without dilution. The capillary electrophoresis method used is outlined in Figure 3.

Figure S- 7 Analysis of anions extracted from post-blast residues resulting from an improvised explosive mixture containing sodium chlorate and sugar.

Conditions: The post-blast residue aqueous sa mple was injected without dilution. The capillary electrophoresis method used is outlined in Figure 2.

Figure S- 8 Analysis of cations extracted from post-blast residues resulting from an improvised explosive mixture containing sodium chlorate and sugar.

Conditions: The post-blast residue aqueous sa mple was injected without dilution. The capillary electrophoresis method used is outlined in Figure 3.

Figure S- 9 Determination of cations in ANFO post-blast residues performed immediately after detonation of the explosive device by the Australian Bomb Data Centre. The portable instrumentation was set up in a mobile laboratory on the munition range and was powered using a diesel generator.

Conditions: The post-blast residue, dissolved in $3 \mathrm{~mL}$ Milli-Q, was spiked into methanol (1:20 dilution) before injection. The capillary electrophoresis method used is outlined in Figure 3. The ambient temperature was $7^{\circ} \mathrm{C}$. 


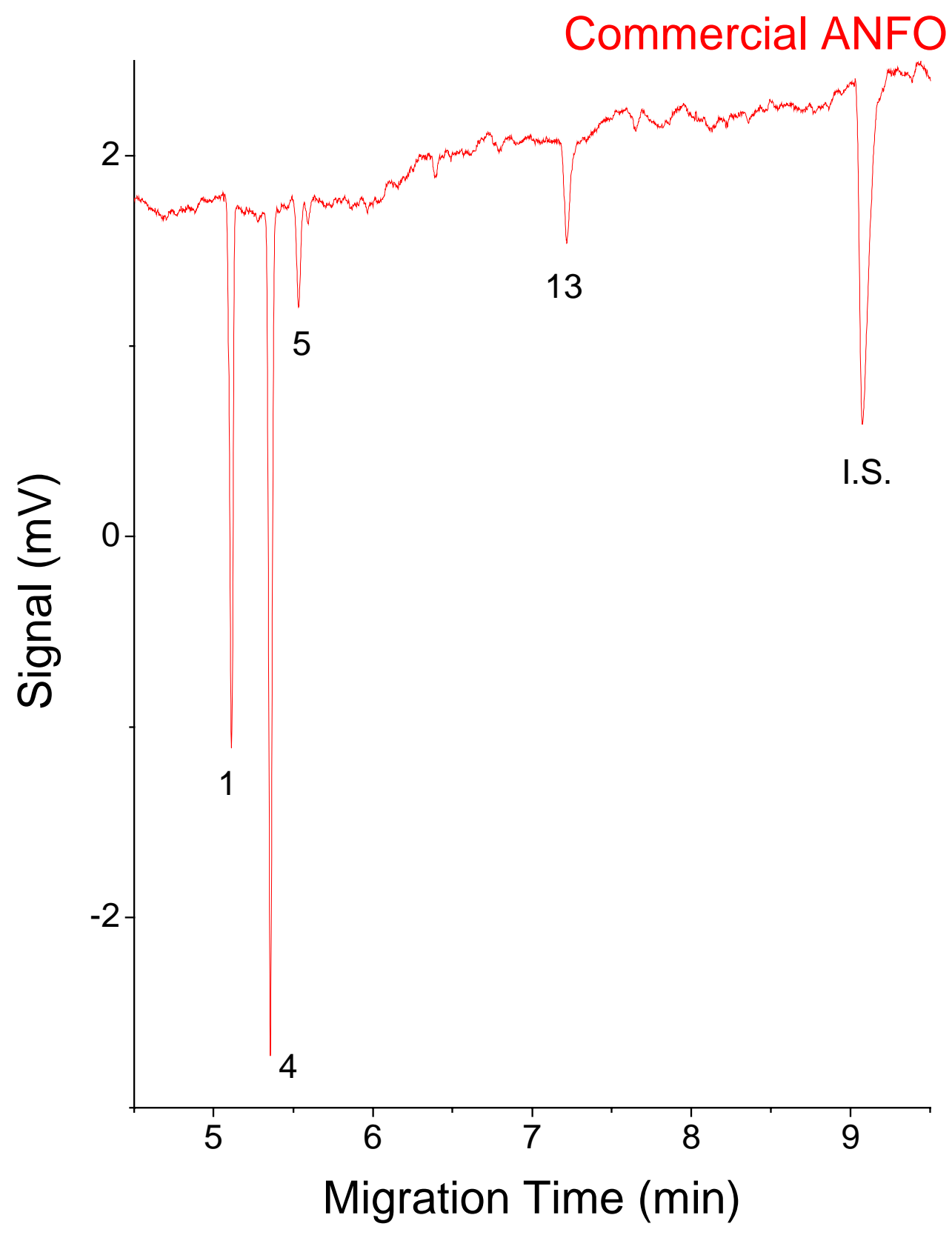

Figure S- 1 


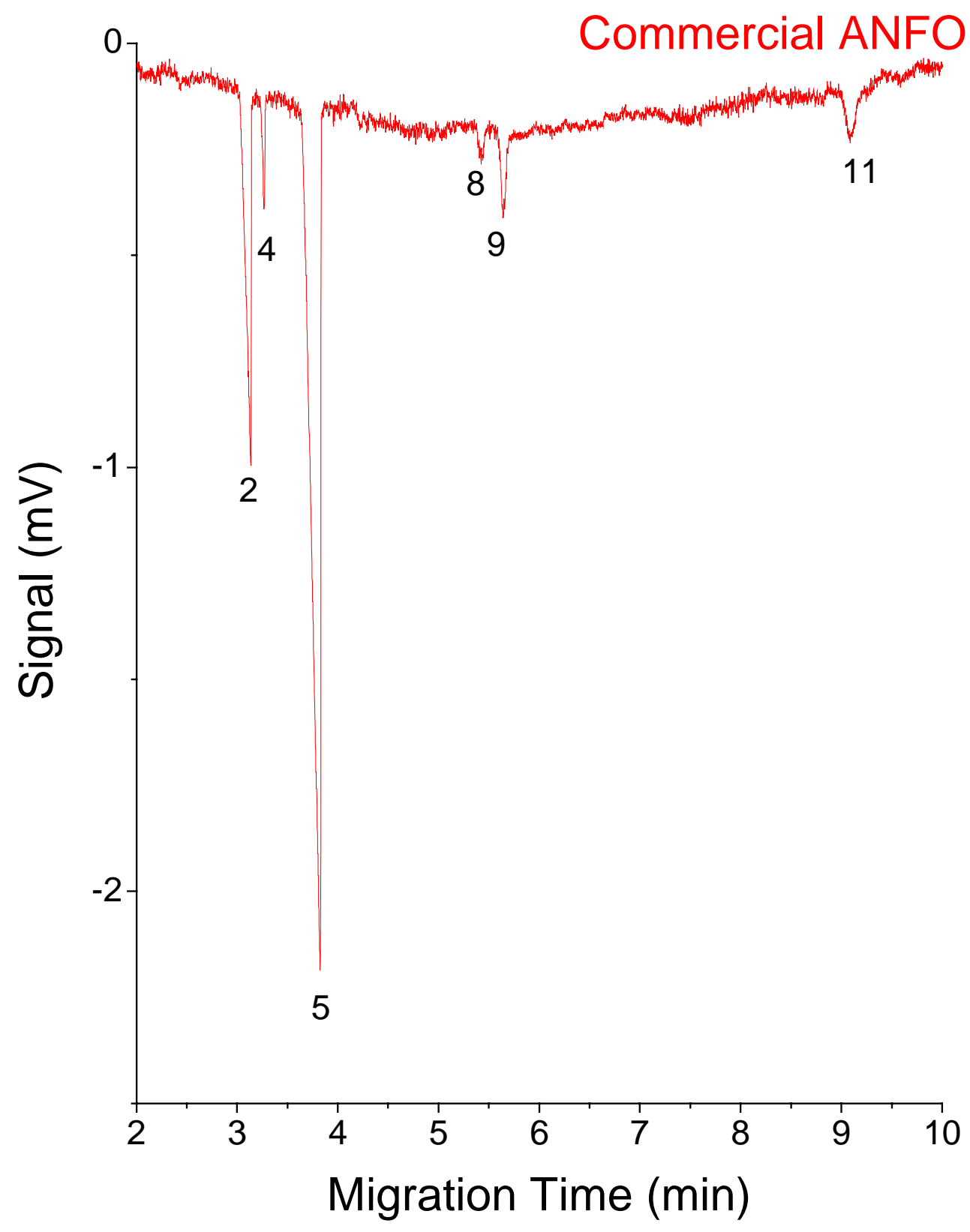

Figure S- 2 


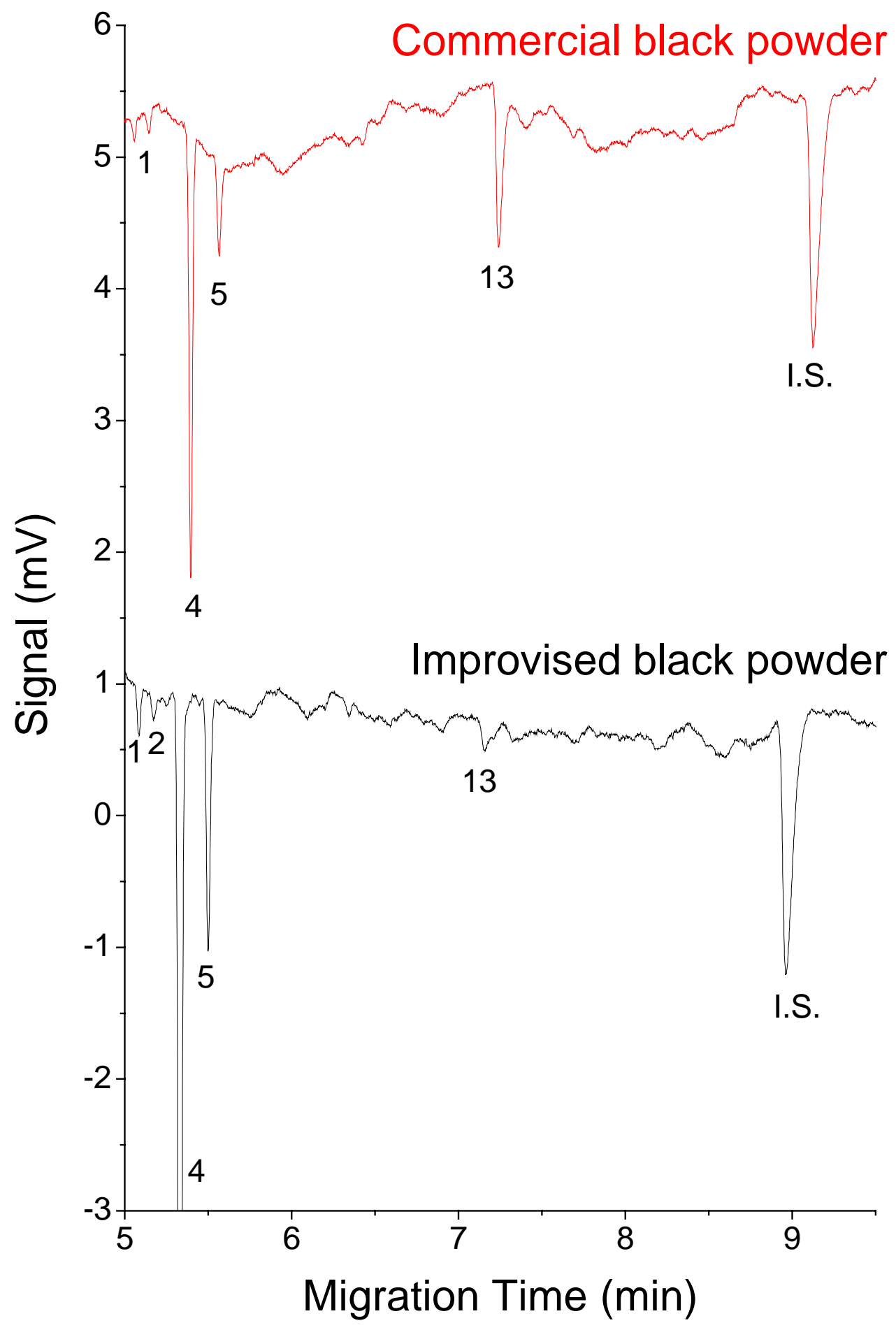

Figure S- 3 


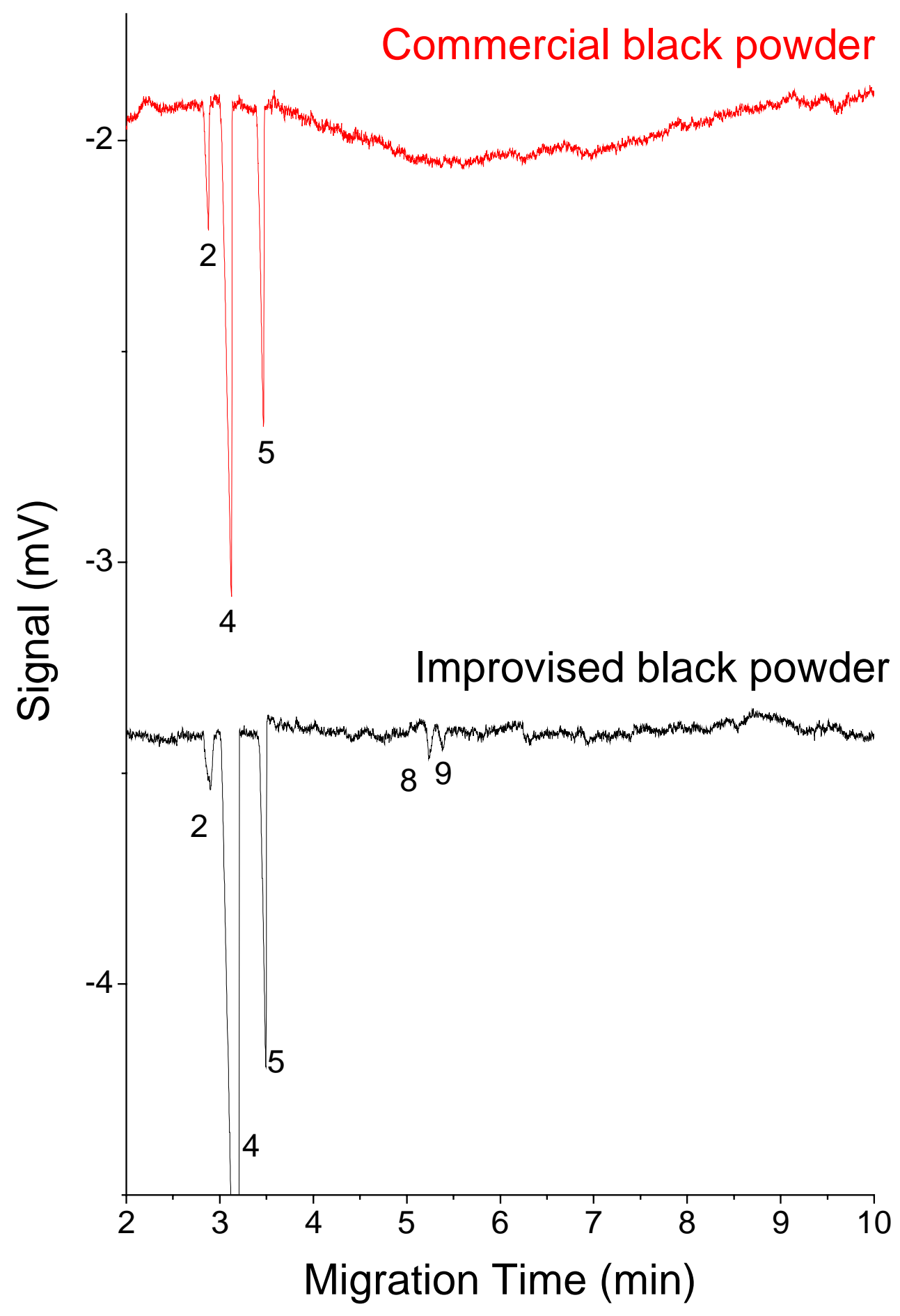

Figure S- 4 


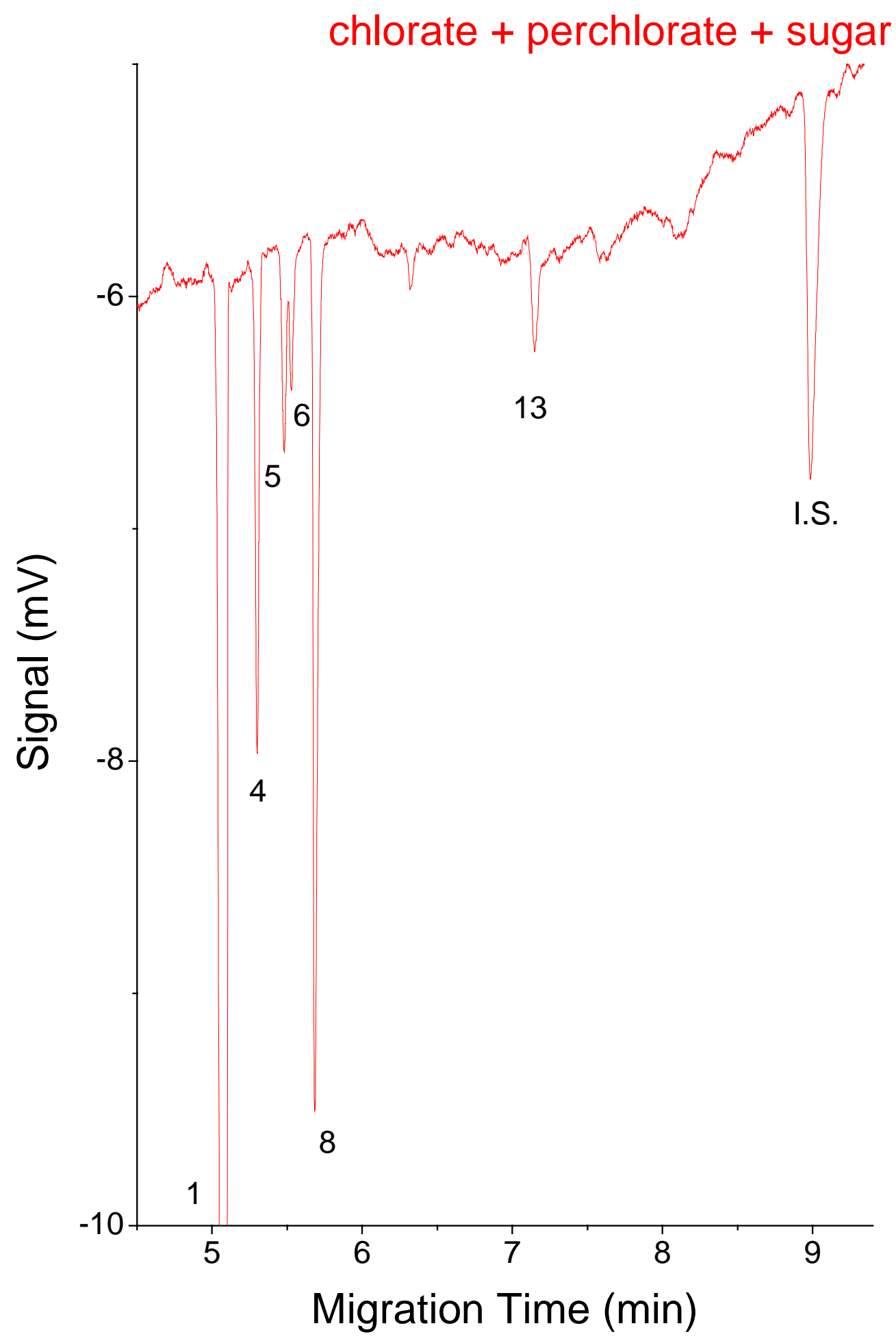

Figure S- 5 


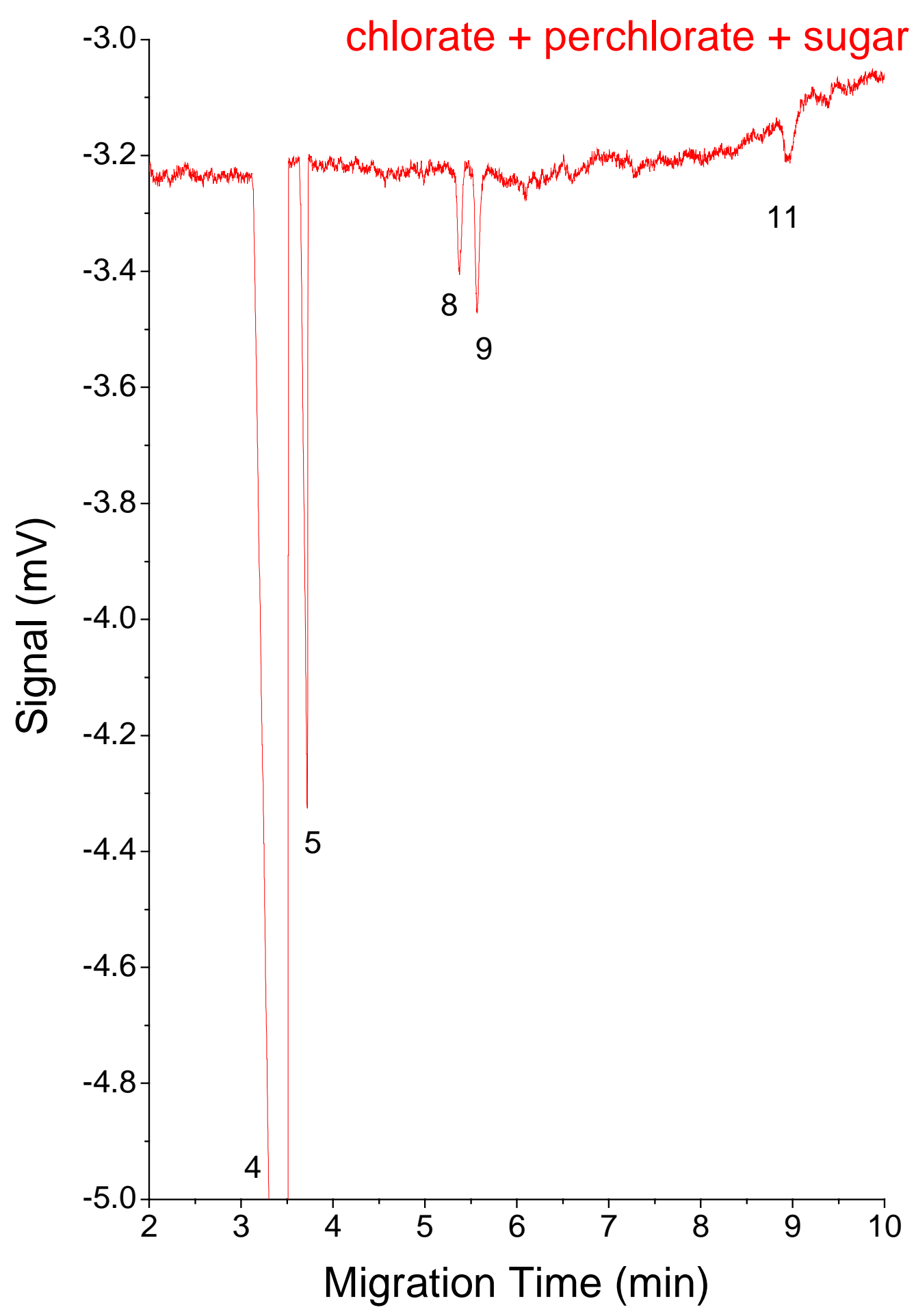

Figure S- 6 
S-10

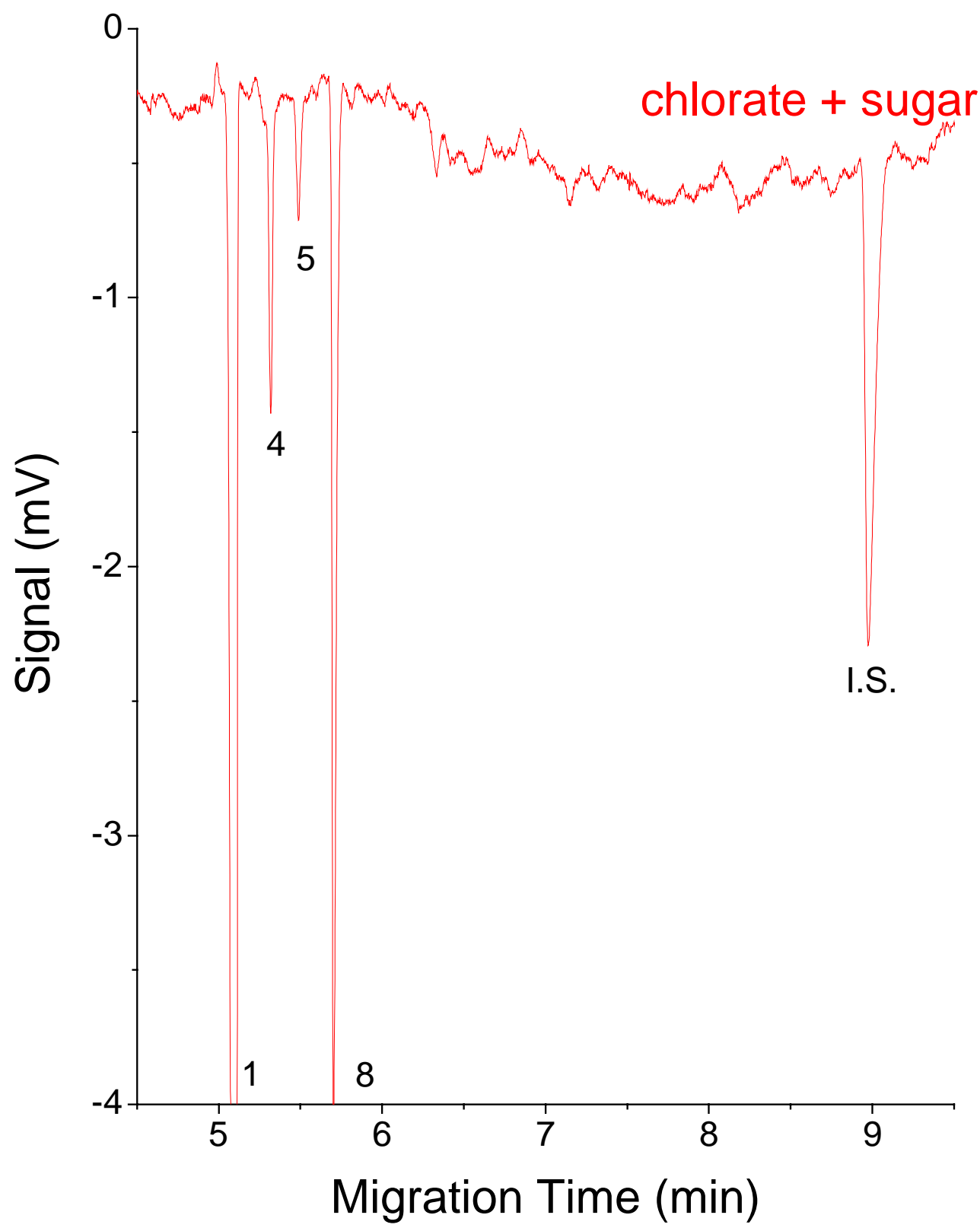

Figure S- 7 
S-11

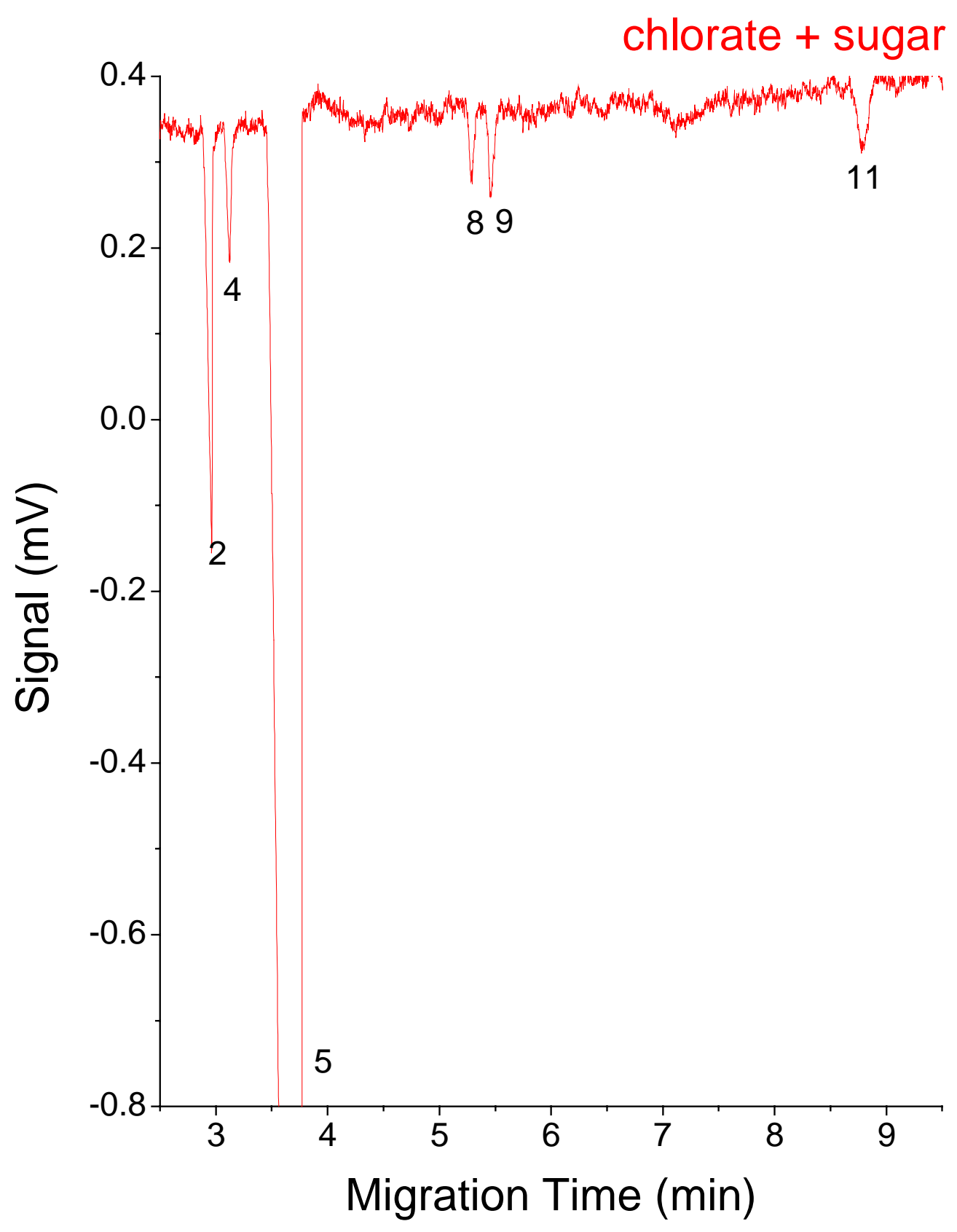

Figure S- 8 
S-12

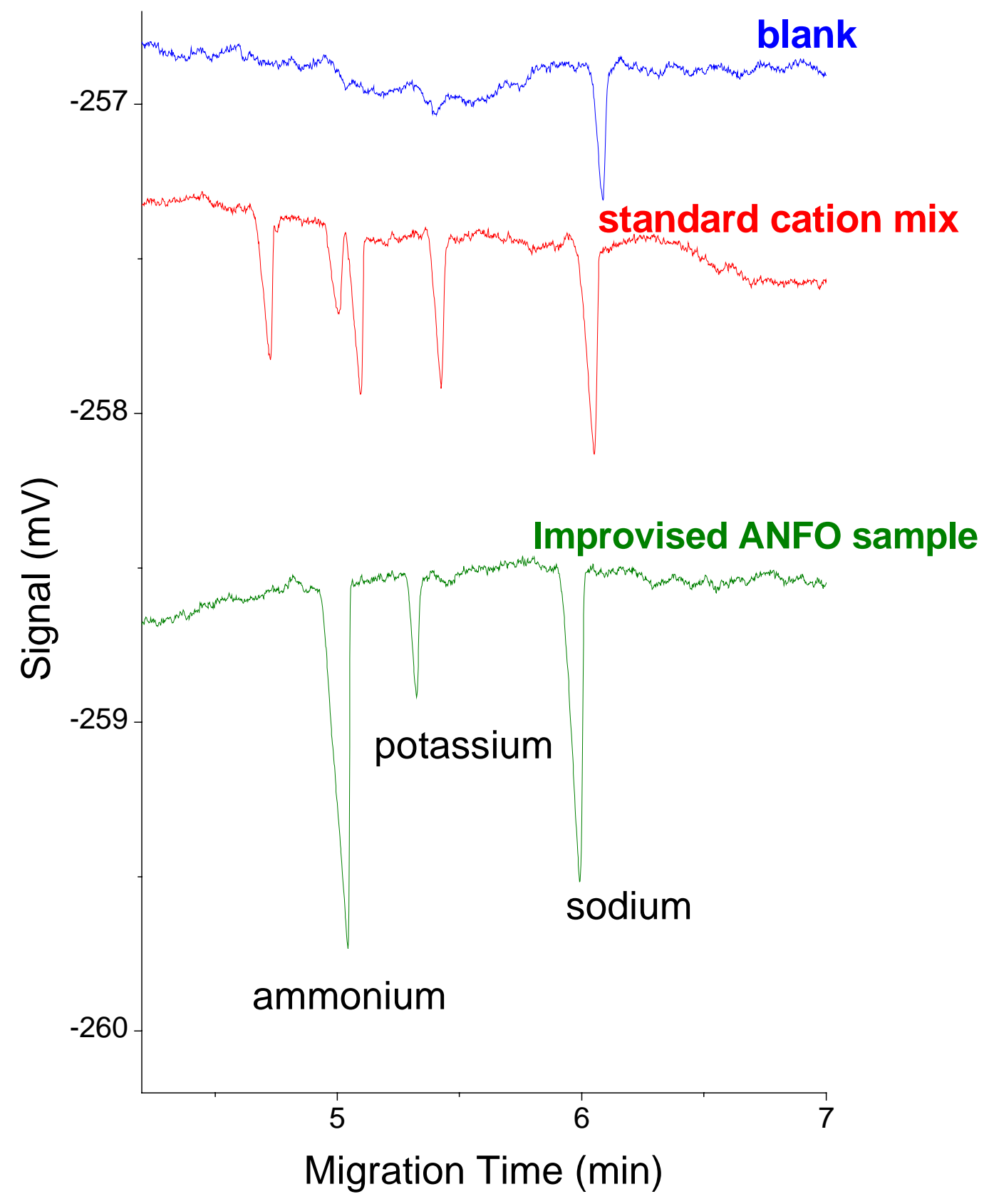

Figure S- 9 